Arq. Bras. Med. Vet. Zootec., v.68, n.4, p.958-966, 2016

\title{
Análise da variabilidade da frequência cardíaca em cordeiros da raça Bergamácia, do nascimento aos 35 dias de idade
}

\author{
[Analysis of heart rate variability in Bergamasca newborn lambs \\ from birth at $35^{\text {th }}$ days of age] \\ K. Koether ${ }^{1}$, M.L.G. Lourenço ${ }^{1 *}$, C.M.V. Ulian $^{1}$, R.S. Gonçalves ${ }^{2}$, M.J. Sudano ${ }^{3}$, \\ R.K.S. Cruz ${ }^{1}$, E.R. Siqueira ${ }^{1}$, A.F. Velo ${ }^{4}$, S.B. Chiacchio ${ }^{1}$ \\ ${ }^{1}$ Universidade Estadual Paulista - Faculdade de Medicina Veterinária e Zootecnia - Unesp - Botucatu, SP \\ ${ }^{2}$ Universidade Estadual Paulista - Faculdade de Medicina - Unesp - Botucatu, SP \\ ${ }^{3}$ Universidade Federal do Pampa - Unipampa - Uruguaiana, RS \\ ${ }^{4}$ Universidade de São Paulo - Instituto de Pesquisas Energéticas e Nucleares - USP - São Paulo, SP
}

\begin{abstract}
RESUMO
A atividade do sistema nervoso autônomo sobre o coração pode ser verificada pela variabilidade da frequência cardíaca (VFC), método que quantifica e assim infere sobre a modulação autonômica cardíaca, refletindo o funcionamento do sistema nervoso autônomo. Durante o período neonatal, diferenças significativas no que se refere à maturação autonômica são descritas em diversas espécies. Embora a espécie ovina venha sendo utilizada como modelo experimental em diversos protocolos em neonatologia e cardiologia em humanos, estudos descritivos sobre a VFC utilizando animais saudáveis e não sedados são escassos na literatura. O objetivo do estudo foi descrever a VFC em cordeiros da raça Bergamácia durante os primeiros 35 dias de idade. Foram realizados exames eletrocardiográficos em 20 cordeiros da raça Bergamácia a partir do nascimento e semanalmente até o $35^{\circ}$ dia de idade. A VFC foi analisada pelo intervalo RR normal (batimentos sinusais), pela frequência cardíaca, pelo índice de tônus vasovagal (iTVV), pela raiz quadrada da média do quadrado das diferenças entre intervalos RR normais adjacentes (RMSSD) e pela raiz quadrada da somatória do quadrado das diferenças dos valores individuais em relação ao valor médio, dividido pelo número de iR-R em 90 segundos - VFC global (RMSM). Todos os parâmetros se alteraram ao longo das semanas. A frequência cardíaca média elevou-se entre o nascimento e os primeiros sete dias de idade, com decréscimo nas quatro semanas subsequentes, sendo o menor valor encontrado aos 35 dias de idade $(145,63 \pm 37,80 \mathrm{bpm})$. Entre 21, 28 e 35 dias de idade, o iTVV elevou-se significativamente, o RMSM a partir do $28^{\circ}$ dia, e, aos 35 dias, o RMSSD, reflexo da ativação parassimpática, exibiu diferenças em relação aos momentos subsequentes. $\mathrm{O}$ início da predominância parassimpática, refletida nos índices da VFC, particularmente o iTVV, ocorre aos 21 dias de idade. A partir dos 35 dias de idade, os índices RMSM e RMSSD podem ser utilizados como marcadores fidedignos das mudanças nos efeitos simpático e parassimpático sobre o coração de cordeiros.
\end{abstract}

Palavras-chave: ovinos, frequência cardíaca, neonatos, controle autonômico

\begin{abstract}
Autonomic nervous system activity can be checked by heart rate variability (HRV), method that quantifies and thus infers on cardiac autonomic modulation, reflecting the functioning of the autonomic nervous system. During the neonatal period significant differences as regards the autonomic maturation, are described in various species. Although the sheep come being used as experimental model of several protocols in neonatology and cardiology in humans, descriptive studies about the VFC using healthy animals and not sedated are scarce in the literature. The objective of this study was to describe the HRV in the Bergamasca race lambs during the first 35 days of age. Electrocardiographic examinations were performed in 20 lambs of the Bergamasca race from birth, in the $7^{\text {th }}, 14^{\text {th }}, 21^{\text {st }}, 28^{\text {th }}$, and $35^{\text {th }}$ days of age.
\end{abstract}

Recebido em 2 de julho de 2014

Aceito em 11 de fevereiro de 2016

*Autor para correspondência (corresponding author)

E-mail: mege@uol.com.br 
The HRV was analyzed by normal RR interval (sinus rate), heart rate, index of vagal tone, and the cardiovascular manifestations (square root of the average of the square of the differences between normal RR intervals adjacent). All parameters have changed over the weeks. The average heart rate increased between the birth and the first seven days of age, with decrease in the subsequent four weeks, being the smallest value found at 35 days of age (145.63 $\pm 37.80 \mathrm{bpm})$. Between 21, 28 and 35 days of age, the iTVV increased significantly, the RMSM from the 28 day, and 35 days, the cardiovascular manifestations, reflex parasympathetic activation, exhibited differences in relation to subsequent times. The beginning of parasympathetic dominance, reflected in the HRV indices, particularly the iTVV, occurs at 21 days of age. From the 35 days of age, the RMSM indices and RMSSD can be used as reliable markers of change in sympathetic and parasympathetic effect on the heart of lambs.

Keyword: ovine, heart rate, neonate, autonomic control

\section{INTRODUÇÃO}

A espécie ovina tem sido amplamente utilizada em diversos protocolos experimentais, em medicina, como modelo para estudo do remodelamento em insuficiência cardíaca crônica em cardiologia (Huang et al., 2004; Borenstein et al., 2006), na eletrocardiografia fetal em obstetrícia (Van Wijngaarden et al., 1996; Wibbens et al., 2005) e em variados protocolos com fármacos em anestesiologia (Clutton et al., 2008). Entre as vantagens em se utilizar a espécie em pesquisa, incluem-se a disponibilidade, o tamanho e o baixo custo de manutenção.

Em medicina veterinária, vale ressaltar que os estudos com ovinos envolvem a ingestão de plantas tóxicas e de outros produtos, como o fluoracetato de sódio. A escassez de dados sobre os valores eletrocardiográficos de referência para diversas raças, entretanto, dificulta a comparação entre os resultados (Schultz et al., 1982; Lago et al., 2009; Kant et al., 2010).

O estudo da variabilidade cardíaca em ovinos tem tido grande interesse, particularmente devido à similaridade, em muitos aspectos, do coração dessa espécie com o dos humanos, incluindo as dimensões das câmaras cardíacas, anatomia coronariana e magnitude das variáveis hemodinâmicas, como pressão arterial, frequência e débito cardíaco (Markovitz et al., 1989; Von Borell et al., 2007). Além disso, a inervação autonômica do coração em ovinos também é semelhante à da espécie humana (Patural et al, 2010; Yiallourou et al., 2013). De acordo com a literatura (Siimes et al., 1984; Siimes et al., 1986; Siimes et al., 1990), as semelhanças entre as duas espécies justificam as pesquisas na espécie ovina, principalmente durante os períodos fetal e neonatal.

Embora sejam fundamentais, estudos com o uso da eletrocardiografia dinâmica de maneira sistemática, em cordeiros não anestesiados, durante o período neonatal, não foram ainda descritos. Grande parte dos estudos envolve procedimentos invasivos, que necessitam de anestesia (Patural et al., 2010; Beuchée et al., 2012), e podem não refletir as particularidades eletrofisiológicas cardíacas nessa faixa etária na espécie ovina.

Visando obter dados que serão úteis para as diversas áreas da veterinária e da medicina que utilizam a espécie ovina como modelo experimental, o presente estudo teve por objetivo descrever a análise da variabilidade da frequência cardíaca no traçado eletrocardiográfico em cordeiros recém-nascidos saudáveis da raça Bergamácia, a partir do nascimento, semanalmente até o $35^{\circ}$ dia de idade, avaliando-se sua evolução durante esse período. Os resultados também poderão ser úteis na rotina clínica dos hospitais veterinários.

\section{MATERIAL E MÉTODOS}

$\mathrm{O}$ estudo foi conduzido na área de Produção e Pesquisa de Leite Ovino da Fazenda Experimental Edgárdia, circunscrita nas coordenadas geográficas $22^{\circ} 47^{\prime} 30^{\prime \prime}$ a $22^{\circ} 50^{\prime}$ de latitude S, e $48^{\circ} 26^{\prime} 15^{\prime \prime}$ a $48^{\circ} 22^{\prime} 30^{\prime \prime}$ de longitude, situada no município de Botucatu, pertencente à Faculdade de Medicina Veterinária e Zootecnia da Unesp, no estado de São Paulo. O estudo foi aprovado pela Comissão de Ética no Uso de Animais - CEUA, sob protocolo número 78/2012. 
Foram colocadas em estação de monta 20 ovelhas da raça Bergamácia, em idade reprodutiva (14 a 16 meses), nulíparas, com escore de condição corporal (ECC) entre 3,0 e 4,0 (considerando 1,0 para animal caquético e 5,0 para obeso). As ovelhas foram acompanhadas mediante exames ultrassonográficos, totalizando dois por fêmea, distribuídos na gestação para garantir um número mínimo de fêmeas gestantes. A partir do terço final de gestação, para facilitar o acompanhamento dos partos, as fêmeas foram confinadas em instalação coberta e com cortinas móveis, separadas em baias para dois $\left(2,0 \times 2,0 \mathrm{~m}^{2}\right)$ ou quatro animais $\left(3,0 \times 2,0 \mathrm{~m}^{2}\right)$, forradas com cama de bagaço de cana com cocho de alimentação e suplementação mineral, e bebedouro automático.

As ovelhas, durante a gestação, foram mantidas em pastagem de Panicum maximum cv. Tanzânia durante o dia; à noite receberam suplementação de silagem de milho e concentrado na proporção de 40\%:60\% (500g/ cabeça / dia de concentrado comercial para ovinos, com $18 \%$ PB), segundo as exigências do NRC (2008), para a referida fase. Após o parto e dentro do barracão, passaram a receber ração para o período de lactação, silagem de milho de fabricação própria, feno de braquiária triturado, sal mineral para ovinos e água ad libitum. Os recém-nascidos permaneceram com suas mães durante todo o período experimental. Durante o estudo, foram feitos exames clínicos periódicos nas fêmeas, controle de endoparasitas por meio de vermifugação mensal dependente do OPG (ovos por grama) e vacinação contra clostridiose.

Os cordeiros receberam atenção especial, para que fosse possível a condução do estudo. Após o nascimento, foram examinados, analisando-se a respiração, o vigor, a atitude, a tentativa e o sucesso de ficar em estação, a atenção em procurar o úbere e o estabelecimento do vínculo materno-neonatal. Os recém-nascidos foram pesados em balança digital, e aferidas temperatura retal, frequência cardíaca e respiratória para acompanhamento clínico e prevenção de intercorrências. A cura do umbigo, com tintura de iodo a $10 \%$, foi realizada uma a duas horas após o nascimento, permitindo a formação da ligação materno-neonatal, para evitar que o cheiro do iodo associado à separação momentânea da mãe levasse à rejeição. Após o nascimento, respeitando-se esse período, os cordeiros foram retirados das mães para pesagem e realização dos exames eletrocardiográficos.

Para a realização do eletrocardiograma, foi empregado aparelho computadorizado (ECG PCTEB, Tecnologia Eletrônica Brasileira, São Paulo, Brasil) nas seis derivações de membros (I, II, III, aVR, aVL e aVF), na velocidade de $50 \mathrm{~mm} /$ segundo e com sensibilidade ajustada para $1 \mathrm{~cm}=1 \mathrm{mV}$, ao longo de um minuto. $\mathrm{O}$ registro foi feito com os animais contidos em local dotado de piso revestido com placas de borracha, de modo a evitar interferências no traçado eletrocardiográfico. Os animais foram mantidos em decúbito lateral direito, sem qualquer tipo de sedação, tranquilização ou anestesia, e o eletrocardiograma foi realizado durante, no mínimo, dois minutos.

Para cada registro eletrocardiográfico, avaliou-se a frequência cardíaca média, a duração do intervalo RR e a VFC no domínio da frequência, por meio da análise de intervalos $R R$ consecutivos (em milissegundos), a partir dos quais se calculou a variância amostral, onde o logaritmo natural (neperiano), representa o índice de tônus vasovagal (iTVV) (Häggström et al., 1996; Tárraga, 2000; Carareto et al., 2007), o RMSSD (raiz quadrada da média do quadrado das diferenças entre intervalos $\mathrm{RR}$ normais adjacentes) e o RMSM (raiz quadrada da somatória do quadrado das diferenças dos valores individuais em relação ao valor médio, dividido pelo número de iR-R em 90 segundos VFC global) (Siimes et al., 1990). A escolha desses índices foi feita por tratar-se de indicadores sensíveis e convencionalmente aceitos para caracterização da VFC (Task Force, 1996) (Fig. 1).

$$
i T V V=\log _{e}\left(\frac{N \sum_{i=1}^{N} R R^{2}{ }_{i}-\left(\sum_{i=1}^{N} R R_{i}\right)^{2}}{N(N-1)} \quad R M S S D=\sqrt{\frac{\sum_{i=1}^{N-1}\left(R R_{i}-R R_{i+1}\right)^{2}}{N-1}} \quad R M S M=\sqrt{\frac{\sum_{i=1}^{N}\left(R R_{i}-\overline{R R}\right)^{2}}{N}}\right.
$$

Figura 1. Fórmulas utilizadas para o cálculo da VFC $\left(\sum=\right.$ somatória; $R R=$ intervalos $R R ; N=$ números de intervalos RR na série selecionada; $\log _{\mathrm{e}}=$ logaritmo natural neperiano). 
Os dados foram analisados utilizando-se o software SAS 9.2 (Statistical Analysis System SAS, SAS Institute, USA) pelo teste de Friedman para se detectar o possível efeito do tempo (momentos) em cada sexo e pelo teste de Mann-Whitney para análise do efeito do tempo sobre cada parâmetro analisado. Utilizou-se também o teste de regressão linear simples para se determinar a dependência das variáveis frequência cardíaca, intervalo RR e VFC (iTVV, RMSSD, RMSM) com a idade e a área de superfície corporal. Para todas as análises, o nível de significância adotado foi de 5\%.

\section{RESULTADOS}

Não se observou diferença significativa entre os sexos, em nenhum momento da análise, não havendo, portanto, nas condições em que o estudo foi realizado, diferenças entre machos e fêmeas $(\mathrm{P}>0,05)$. Houve efeito do tempo sobre os parâmetros FC, iTVV, RMSSD e RMSM, em diferentes momentos.

Em relação ao comportamento da frequência cardíaca média, ocorreu elevação entre o nascimento e os primeiros sete dias de idade, com decréscimo nas quatro semanas subsequentes, sendo o menor valor encontrado aos 35 dias de idade (145,63 $\pm 37,80 \mathrm{bpm})$. A análise da frequência cardíaca versus a idade é demonstrada na Fig. 2. A análise de regressão linear demonstrou a redução da frequência cardíaca em decorrência da evolução da idade (Fig. 3).

$\mathrm{Na}$ análise da VFC no domínio do tempo, foram utilizados os parâmetros RR médio, o índice de tônus vagal e a raiz quadrada da média do quadrado dos intervalos RR (RMSSD) para estimar a variabilidade dos intervalos RR em período curto de tempo (atividade parassimpática) e a raiz quadrada da somatória do quadrado das diferenças dos valores individuais em relação ao valor médio, dividido pelo número de iR-R em 90 segundos (RMSM), como índice expressivo da VFC total ou global (atividade simpática e parassimpática).

No que se refere ao intervalo RR médio, constatou-se diferença estatística a partir da terceira semana de vida, com elevação significativa nas duas semanas subsequentes, aos 28 e 35 dias de idade, respectivamente. Durante as primeiras três semanas, a média dos intervalos manteve-se estável, não ocorrendo diferença entre as semanas (Tab. 1; Fig. 2). O intervalo RR acresceu à medida que a idade dos cordeiros aumentou, como pode ser observado na Fig. 2.

Tabela 1. Média e desvio-padrão da frequência cardíaca, do intervalo RR e dos índices da variabilidade da frequência cardíaca pelo eletrocardiograma (iTV, RMSSD e RMSM) em cordeiros neonatos, do nascimento aos 35 dias de idade

\begin{tabular}{cccccc}
\hline Idade & FC $(\mathrm{bpm})$ & $\begin{array}{c}\text { RR intervalo } \\
(\mathrm{ms})\end{array}$ & iTVV & RMSSD & RMSM \\
\hline Nascimento & $191,955 \pm 42,176 \mathrm{ac}$ & $334,864 \pm 69,155 \mathrm{a}$ & $5,017 \pm 0,172 \mathrm{ac}$ & $15,292 \pm 7,701 \mathrm{a}$ & $20,975 \pm 10,248 \mathrm{a}$ \\
7 dias & $214,955 \pm 54,582 \mathrm{~b}$ & $297,045 \pm 69,125 \mathrm{a}$ & $4,907 \pm 0,226 \mathrm{~b}$ & $21,934 \pm 31,928 \mathrm{ab}$ & $26,025 \pm 19,090 \mathrm{a}$ \\
14 dias & $195,500 \pm 37,744 \mathrm{ab}$ & $305,909 \pm 57,223 \mathrm{a}$ & $4,977 \pm 0,166 \mathrm{ab}$ & $29,335 \pm 41,033 \mathrm{~b}$ & $29,761 \pm 24,754 \mathrm{ab}$ \\
21 dias & $173,045 \pm 30,724 \mathrm{~cd}$ & $361,182 \pm 56,174 \mathrm{~d}$ & $5,072 \pm 0,104 \mathrm{c}$ & $24,810 \pm 16,406 \mathrm{ab}$ & $27,972 \pm 12,433 \mathrm{a}$ \\
28 dias & $158,818 \pm 22,142 \mathrm{de}$ & $379,818 \pm 44,889 \mathrm{~b}$ & $5,152 \pm 0,106 \mathrm{~d}$ & $33,808 \pm 30,843 \mathrm{~b}$ & $38,565 \pm 23,997 \mathrm{~b}$ \\
35 dias & $145,636 \pm 37,803 \mathrm{e}$ & $441,636 \pm 115,282 \mathrm{c}$ & $5,267 \pm 0,208 \mathrm{e}$ & $55,327 \pm 58,147 \mathrm{c}$ & $52,458 \pm 34,841 \mathrm{c}$ \\
\hline
\end{tabular}

(*) Letras sobrescritas na mesma coluna diferem significativamente. $(\mathrm{P}<0,05)$. Valores são expressos sob a forma de média e desvio-padrão.

Os índices da VFC no domínio do tempo, iTVV, RMSSD e RMSM, apresentaram comportamento distinto durante as semanas de análise. Entre o nascimento e a primeira semana de idade, conjuntamente com a elevação da frequência cardíaca, o índice de tônus vasovagal apresentou decréscimo significativo, não havendo, contudo, alteração significativa nos índices RMSSD e RMSM até o $21^{\circ}$ dia de idade. Entre os 14 e 21 dias, a diminuição significativa da frequência cardíaca também foi notada em concomitância ao acréscimo do iTVV. 


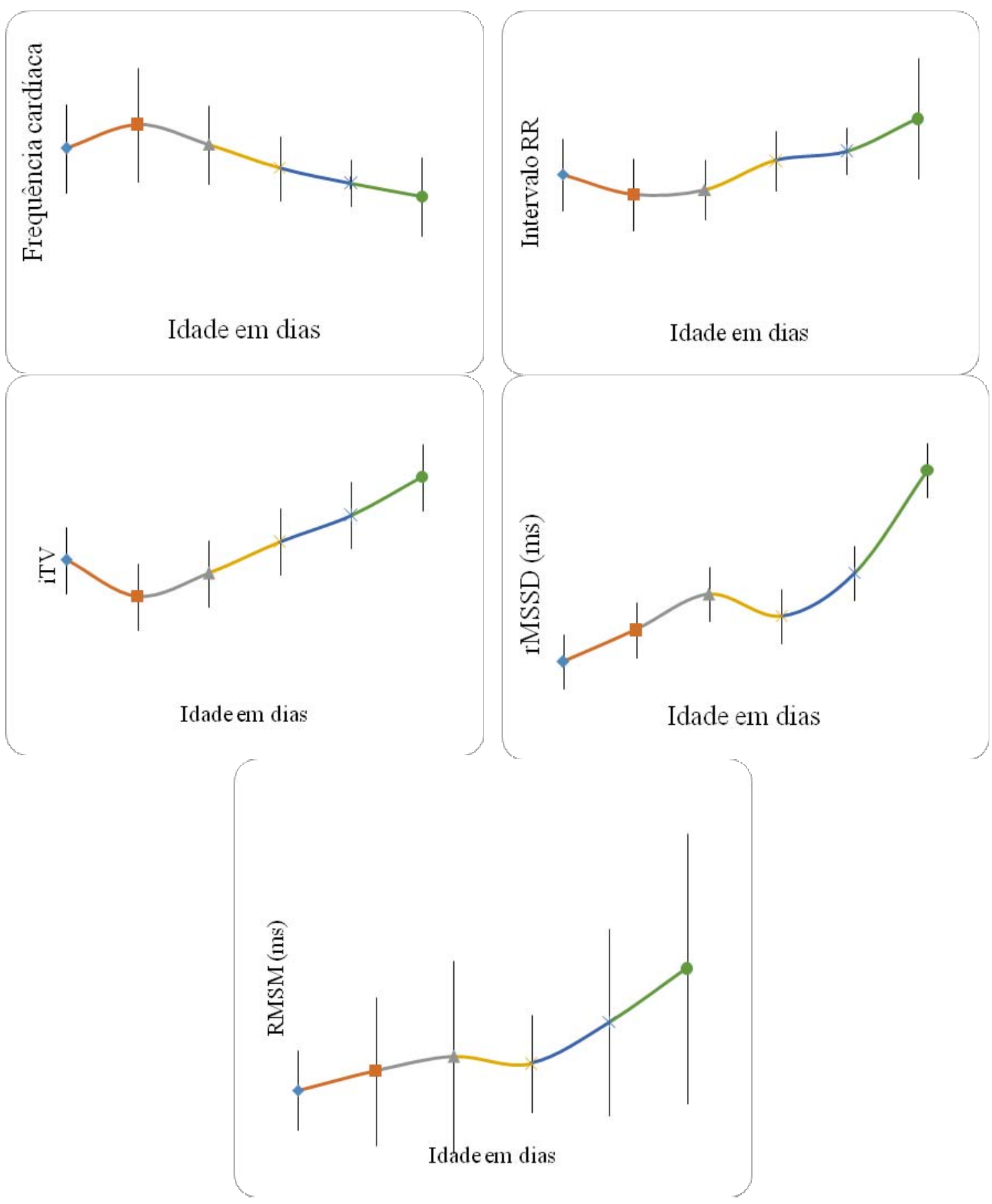

Figura 2. Evolução da frequência cardíaca, intervalo RR, iTVV, RMSSD e RMSM em cordeiros neonatos ao longo dos 35 dias de idade.

Entre 21, 28 e 35 dias de idade, o iTVV elevouse significativamente, o RMSM a partir do $28^{\circ}$ dia, e, aos 35 dias, os três índices se elevaram estatisticamente quando comparados às semanas anteriores. Segundo as análises de regressão linear, houve dependência entre os índices RMSSD e RMSM e a idade dos cordeiros $(\mathrm{P}<0,05)$ (Fig. 3). 


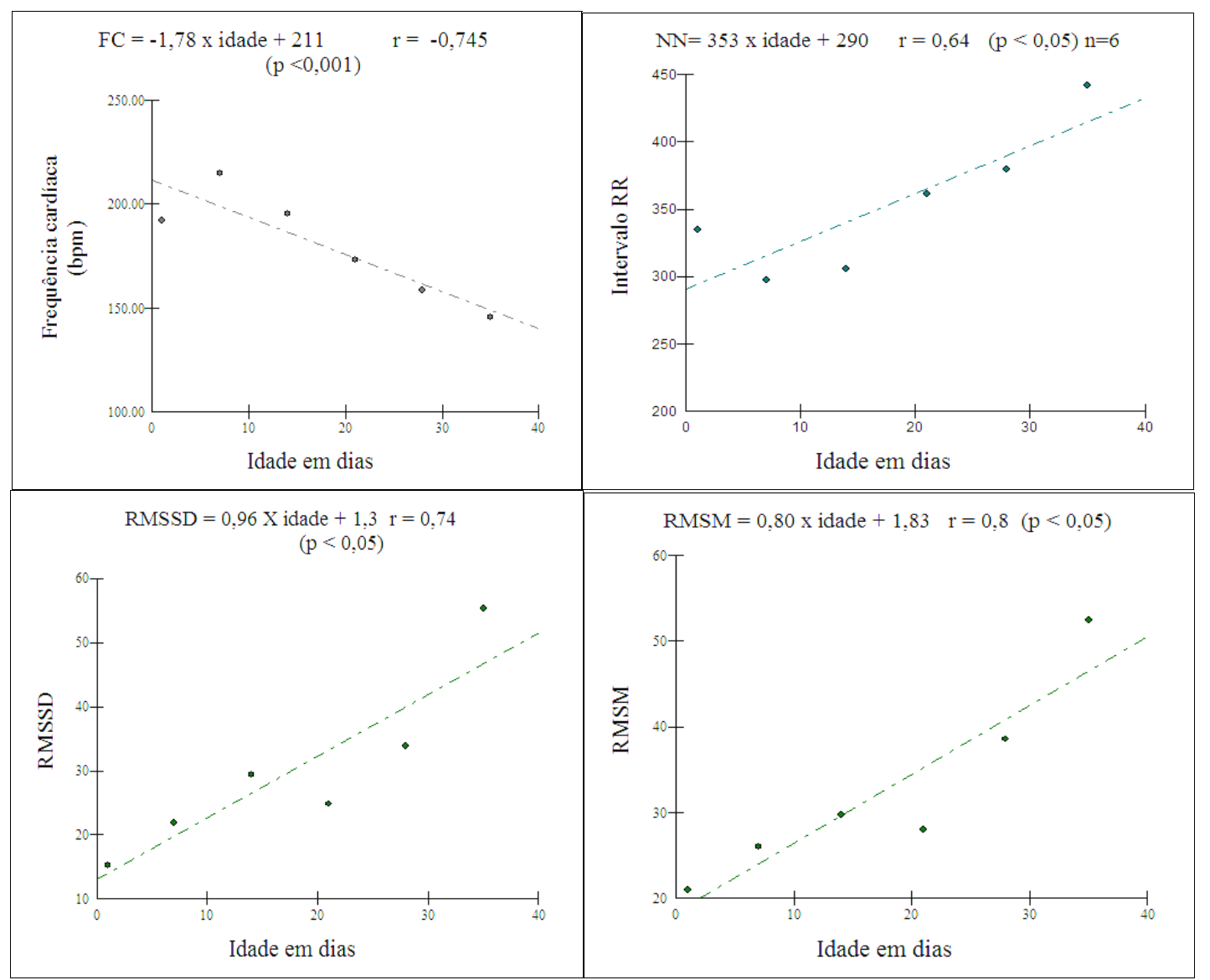

Figura 3. Frequência cardíaca, intervalo RR, RMSSD e RMSM em função da idade, utilizando-se análise de regressão linear.

\section{DISCUSSÃO}

O sistema nervoso autônomo exerce um papel fundamental na modulação da função cardíaca, não somente sob condições fisiológicas, como também nas alterações cardíacas. A homeostase orgânica em diversas situações cotidianas é mantida por meio do equilíbrio entre o sistema simpático e o parassimpático, tendo por base o conceito de antagonismo entre eles. Se, por um lado, o automatismo cardíaco se caracteriza pela despolarização involuntária, o controle da frequência de despolarização é modulado pelo sistema nervoso autônomo (Tárraga, 2000; Carareto et al., 2007).

Sob influência autonômica, a frequência de despolarização cardíaca se alterna periodicamente, sendo definida, portanto, como a variabilidade da frequência cardíaca. A idade exerce grande influência sobre essa variabilidade, reflexo do balanço entre os sistemas simpático e parassimpático. Quanto maior a idade, menor a variabilidade da frequência cardíaca, sendo ela menor em idosos, quando comparados aos adultos jovens. Contudo, essa relação sofre uma inversão quando se trata de recém-nascidos, que possuem menor VFC devido à imaturidade do sistema nervoso autônomo (Heier et al., 2010; Seling et al., 2011)

O objetivo deste estudo foi descrever a análise da variabilidade da frequência cardíaca neonatal em cordeiros, avaliando-se seu comportamento durante o período neonatal, considerado pelos primeiros 30 dias de idade. Nas primeiras 24 horas (nascimento), a frequência cardíaca apresentou-se elevada, superior aos valores descritos para animais adultos da espécie ovina (90-115bpm) (Feitosa e Benesi, 2014) e também para cordeiros na mesma faixa etária (Bovino, 2011; Avila, 2013; Ulian et al., 2014). Neonatos de diferentes espécies apresentam FC elevada 
(Feitosa e Benesi, 2014), sendo esta uma compensação ao menor volume sistólico e resistência vascular periférica durante o período neonatal (Grundy, 2006). Com o passar das semanas, houve decréscimo acentuado, sendo o menor valor encontrado somente aos 35 dias de idade.

Durante o primeiro mês de vida, as oscilações na frequência cardíaca foram provavelmente causadas por ajustes complexos nos mecanismos de controle do sistema nervoso autônomo para adequação do débito cardíaco frente às demandas da circulação periférica do organismo em crescimento (Siimes et al., 1990). O decréscimo da frequência em função da idade, demonstrado na análise de regressão linear do presente estudo (Fig. 3), deixou bem claro que a maturação do sistema nervoso autônomo, no que tange ao controle da frequência de despolarização cardíaca, ultrapassa o período neonatal na espécie ovina, uma vez que os valores ainda se encontravam, aos 35 dias, superiores aos descritos para adultos. A idade foi, portanto, um fator preditor da diminuição da frequência cardíaca em cordeiros neonatos, sendo $74,48 \%$ de seu decréscimo ocorrido em função da idade.

Quanto ao comportamento da VFC em cordeiros neonatos, embora a literatura seja ampla na espécie, grande parte discorre sobre neonatos prematuros ou fetos, não sendo adequada a comparação com os dados obtidos no presente estudo. Sob condições similares, contudo, sem a interferência de bloqueios farmacológicos, os resultados (FC, RR) aqui apresentados foram semelhantes aos descritos por Siimes et al. (1990) para cordeiros com menos de 30 dias de idade, embora maiores em relação aos índices RMSM e RMSSD, que refletem a variabilidade global (simpático e parassimpático) e a atuação parassimpática, respectivamente.

Os índices utilizados apresentaram, no decorrer das semanas, acréscimo inversamente proporcional quando comparados à frequência cardíaca. O domínio simpático predominou nas três primeiras semanas de vida, havendo até esse momento frequência cardíaca elevada sem que os índices RMSM e RMSSD se elevassem significativamente, o que demonstra um forte impacto sobre a VFC, conforme descrito por Von Borrell et al. (2007).
Aos 21 dias de idade, pode-se perceber o início da expressão do sistema parassimpático refletindo-se na elevação do índice de tônus vasovagal, embora ainda insipiente para promover elevação de outros índices, como o RMSM e o RMSSD. O iTVV demonstrou maior sensibilidade em detectar o início da atuação parassimpática durante as primeiras semanas de vida. A partir dos 21 dias, o índice sofreu elevação significativa com o progredir das semanas, indicando início do predomínio vagal. A maior sensibilidade desse índice deveu-se, em termos mais simples, à utilização do logaritmo natural (neperiano), função matemática que tornou possível o estudo de fenômenos que evoluem de maneira exponencial, como a atividade parassimpática. $\mathrm{O}$ prolongamento dos intervalos $R R$, retratado pela diminuição da frequência cardíaca, mais proeminente a partir do $21^{\circ}$ dia, também confirmou o início da atuação autonômica parassimpática cardíaca, também observada na variabilidade da frequência.

O RMSM, índice reflexo da atividade parassimpática, apresentou elevação significativa somente a partir dos 28 dias, demonstrando a imaturidade desse sistema, diferente do RMSSD, indicativo da atividade autonômica global (simpático e parassimpático). Aos 28 dias e aos 35 dias de idade, ambos diferiram significativamente quando comparados ao nascimento, o que demonstra o início do desenvolvimento da predominância vagal ainda no período neonatal, contudo ainda incompleta, uma vez que a frequência cardíaca ainda se encontrava acima dos limites de referência, indicando predomínio simpático.

O controle neural autonômico desenvolve-se de maneira complexa durante o período neonatal, entretanto percebe-se predominância simpática durante as três primeiras semanas de idade, quando, a partir de então, observa-se nitidamente a influência vagal. Durante essa fase do desenvolvimento neonatal, é provável que a indução de bloqueio adrenérgico farmacológico promova oscilações mais sutis nos índices da VFC, quando comparados a cordeiros com mais de 30 dias de idade, uma vez que a atuação parassimpática ainda não se encontra em sua plenitude.

Diferentemente dos adultos, o controle neural autonômico da VFC em cordeiros neonatos 
encontra-se sob influência simpática até as primeiras três semanas de idade, quando, a partir de então, observa-se o início da atuação parassimpática. Uma vez descritos os índices da VFC, que refletem o controle autonômico da adaptação cardiopulmonar perinatal, alterações desses padrões específicos durante o período neonatal poderiam ser utilizados com propósitos diagnósticos ou prognósticos.

\section{CONCLUSÕES}

Com base no presente estudo, concluí-se que o impacto do sistema nervoso simpático sobre a frequência cardíaca diminui com o progredir da idade durante o período neonatal dos cordeiros, dando lugar ao sistema parassimpático, por volta dos 21 dias de idade. Por serem animais de uso experimental para a cardiologia humana e a veterinária, faz-se necessária a definição da fisiologia e dos períodos em que ocorre o maior desenvolvimento cardíaco. Os resultados obtidos podem auxiliar na rotina clínica, evitando perdas na produção durante a fase de cria, e como referência para os protocolos experimentais na medicina.

\section{REFERÊNCIAS}

AVILA, L.G. Avaliação clínico-laboratorial de cordeiros nascidos a termo e prematuros. 2013. 100f. Tese (Doutorado) - Faculdade de Medicina Veterinária, Universidade Estadual Paulista "Júlio de Mesquita Filho", Araçatuba, SP.

BEUCHÉE, A.; HERNANDEZ, A.L.; DUVAREILLE, C. et al. Influence of hypoxia and hypercapnia on sleep state-dependent heart rate variability behavior in newborn lambs. Sleep, v.35, p.1541-1549, 2012.

BORENSTEIN, N.; BRUNEVAL, N.; BEHR, L. et al. An ovine model of chronic heart failure: Echocardiographic and tissue Doppler imaging characterization. J. Cardiac Surg., v.21, p.50-56, 2006.

BOVINO, F. Determinação do escore de Apgar, dos valores hemogasométricos e do proteinograma sérico em cordeiros (Ovis aries) nascidos de partos normaise de cesarianas. 2011. 85f. Dissertação (Mestrado em Ciências Animal) - Faculdade de Medicina Veterinária, Universidade Estadual Paulista "Júlio de Mesquita Filho", Araçatuba, SP.
CARARETO, R.; SOUSA, M.G.; ZACHEU; J.C. et al. Variabilidade da frequência cardíaca em cães anestesiados com infusão contínua de propofol e sufentanil. Arq. Bras. Med. Vet. Zootec., v.59, p.329-323, 2007.

CLUTTON, E.R.; GLASBY, M.A. Cardiovascular and autonomic nervous effects of edrophonium and atropine combinations during neuromuscular blockade antagonism in sheep. Vet. Anaesth. Anal., v.35, p.191-200, 2008.

FEITOSA, F.L.F.; BENESI, F.J. Semiologia de animais recém-nascidos ruminantes e equídeos. In: FEITOSA, F.L.F. (Ed.). Semiologia veterinária, a arte do diagnóstico. São Paulo: Roca, 2014. p.69-95.

GRUNDY S.A. Clinically relevant physiology of the neonate. Vet. Clin. North Am. Small Anim. Pract., v.36, p.443-459, 2006.

HÄGGSTRÖM, J.; HAMLIN, R.L.; HANSSON, K.; KVART, C. Heart rate variability in relation to severity of mitral regurgitation in Cavalier king charles spaniels. J. Small Anim. Pract., v.37, p.69-75, 1996.

HEIER, C.R.; HAMPTON, T.G.; WANG, D.; DiDONATO, C.J. Development intervals growth of FVB/N neonate mice. BMC Physiol., v.10, p.1-9, 2010.

KANT, V.; SRIVASTAVA, A.K.; VERMAPK, R.R.; PANKAJ, N.K. Alterations in electrocardiographic parameters after sub acute exposure of fluoride and ameliorative action of aluminium sulphate in goats. Biol. Trace Elem. Res., v.134, p.188-194, 2010.

LAGO, E.P.; MELO, M.M.; ARAÚJO, R.B. et al. Perfis eletrocardiográficos e ecodopplercardiográfico de ovinos após ingestão suspensa aquosa de Mascagnia rígida Griseb. (Malpighiaceae). Arq. Bras. Med. Vet. Zootec., v.61, p.853-862, 2009.

MARKOVITZ, L.J.; SAVAGE, E.B.; RATCLIFFE, M.B. et al. Large animal model of left ventricular aneurysm. Ann. Thorac. Surg., v.48, p.838-845, 1989.

PATURAL, H.; HILARIE, M.; PICHOT, V.A. et al. Postnatal autonomic activity in the preterm lamb. Res. Vet. Sci., v.89, p.242-249, 2010. 
SCHULTZ, R.A.; COETZER, J.A.; KELLERMAN, T.S.; NAUDÉ, T.W. Observation on clinical, cardiac and histopathology effects of fluoroacetate in sheep. Onderstepoort J. Vet. Res., v.49, p.237-245, 1982.

SELING, F.A.; TONOLLI, E.R.; SILVA, E.V. C.M.; GODOY, M.F. Variabilidade da frequência cardíaca em neonatos prematuros e de termo. Arq. Bras. Cardiol., v.96, p.1-7, 2011.

SIIMES, A.S.I.; VALIMAK,I I.A.T.; SARAJAS, H.S.S. et al. Heart rate variation in relation to age and sleep state in neonatal lambs. Acta Physiol. Scand., v.537, Supp1, p.7-15, 1984.

SIIMES, A.S.I.; VALIMAKI, I.A.T.; ANTILA, K.J. et al. Regulation of heart rate variation by the autonomic nervous system in neonatal lambs. Pediatr. Res., v.27, p.383-391, 1990.

SIIMES, A.S.I.; VALIMAKI, I.A.T.; OJA, R.T.; ANTILA, K.J. Detection of components of autonomic cardiac control by time series analysis of heart rate in lambs. Technical report. In: ROLFE, P. (Ed.). Fetal physiological measurements. London: Butterworths, 1986. p.259-266.

TÁRRAGA, K.M.; SPINOSA, H.S.; CAMACHO, A.A. Electrocardiographic evaluation of two anesthetic combinations in dogs. Arq. Bras. Med. Vet. Zootec., v.52, p.138$143,2000$.
TASK FORCE. Heart rate variability - Standards of measurement, physiological interpretation, and clinical use. Eur. Heart J., v.17, p.354-381, 1996.

ULIAN, C.M.V.; KOETHER, K.; LOURENÇO, M.L.G. et al. Physiological parameters in neonatal lambs of the bergamasca breed. Acta Sci. Vet., v.42, p.1183, 2014.

VAN WIJNGAARDEN, W.J.; HAAN, H.H.; SAHOTA, D.S. et al. Changes in the PR interval-fetal heart rate relationship of the electrocardiogram during fetal compromise in chronically instrumented sheep. Am. J. Obstet. Gynecol., v.75, p.548-554, 1996.

VON BORELL, E.; LANGBEIN, J.; DESPRÉS, $\mathrm{G}$. et al. Heart rate variability as a measure of autonomic regulation of cardiac activity for assessing stress and welfare in farm animals: a review. Physiol. Behav., v.92, p.293-316, 2007.

WIBBENS, B.; WESTGATE, J.A.; BENNET, L. et al. Profound hypotension and associated electrocardiographic changes during prolonged cord occlusion in the near term fetal sheep. Am. J. Obstet. Gynecol., v.193, p.803-810, 2005.

YIALLOUROU, S.R.; WITCOMBE, N.B.; SANDS, S.A. et al. The development of autonomic cardiovascular control is altered by preterm birth. Early Hum. Dev., v.89, p.145-152, 2013. 